Article

\title{
Indoor Fingerprint Positioning Based on Wi-Fi: An Overview
}

\author{
Shixiong Xia ${ }^{1}$, Yi Liu ${ }^{1}$, Guan Yuan ${ }^{1,2, *}$, Mingjun Zhu ${ }^{1}$ and Zhaohui Wang ${ }^{1}$ \\ 1 School of Computer Science and Technology, China University of Mining and Technology, \\ No. 1 Daxue Road, Xuzhou 221116, China; xiasx@cumt.edu.cn (S.X.); jim2954891@163.com (Y.L.); \\ 13912034367@163.com (M.Z.); wzh_tree@163.com (Z.W.) \\ 2 Jiangsu Key Laboratory of Mine Mechanical and Electrical Equipment, \\ China University of Mining and Technology, No. 1 Daxue Road, Xuzhou 221116, China \\ * Correspondence: yuanguan@cumt.edu.cn
}

Academic Editors: Sisi Zlatanova, Kourosh Khoshelham, George Sithole and Wolfgang Kainz Received: 18 February 2017; Accepted: 25 April 2017; Published: 28 April 2017

\begin{abstract}
The widely applied location-based services require a high standard for positioning technology. Currently, outdoor positioning has been a great success; however, indoor positioning technologies are in the early stages of development. Therefore, this paper provides an overview of indoor fingerprint positioning based on Wi-Fi. First, some indoor positioning technologies, especially the Wi-Fi fingerprint indoor positioning technology, are introduced and discussed. Second, some evaluation metrics and influence factors of indoor fingerprint positioning technologies based on Wi-Fi are introduced. Third, methods and algorithms of fingerprint indoor positioning technologies are analyzed, classified, and discussed. Fourth, some widely used assistive positioning technologies are described. Finally, conclusions are drawn and future possible research interests are discussed. It is hoped that this research will serve as a stepping stone for those interested in advancing indoor positioning.
\end{abstract}

Keywords: indoor positioning technology; $\mathrm{Wi}-\mathrm{Fi}$; fingerprint

\section{Introduction}

The wide application of location-based services requires a high standard of positioning technology. Although outdoor positioning technology has matured, research into high accuracy positioning technology has gradually transferred from the outdoors to indoor [1]. This is mainly due to two reasons: first, according to the investigation and analysis in reference [1], people generally spend about $80-90 \%$ of their daily time indoors. Furthermore, $70 \%$ of mobile phones are used indoors and $80 \%$ of data communications are performed indoors. Second, the above-mentioned data show that the demand for indoor mobile communication is very strong. Nevertheless, function areas in indoor spaces-especially large indoor public spaces such as airports, plazas, hotels, and so on-become more complicated and larger, for example, restaurants, cinemas, jewelers and many other kinds of stores may be integrated on only one floor level in a plaza, and so they may prove difficult for customers to find interesting areas. Therefore, from the above-mentioned viewpoints, indoor location-based services are of huge market potential.

During the last decade, indoor positioning technology has rapidly developed, with many new emergent technologies and methods. From the RADAR system developed by Microsoft Research Asia [2] and the Horus system put forward by the University of Maryland [3], to the LIFS system proposed by Tsinghua University [4] and the WHERE@UM system [5] created by University of Minho. As positioning results are becoming more accurate, costs are similarly reduced. 
Indoor positioning technologies can be mainly divided into two fields, one based on a 2D model, and the other based on a 3D model. The positioning technologies based on a 2D model mainly use Bluetooth [6], ZigBee [7], and Wi-Fi as positioning signals, and combine the signal intensity or space-time attributes with positioning algorithms. Infrared [8], ultra-wideband (UWB) [9], and ultrasonic [10] are used for positioning based on a 3D model. Compared with 3D model-based technology, the hardware used in 2D model-based positioning technology is much cheaper than Wi-Fi routers, which can easily bring down costs, and the network it requires has already been widely distributed. Based on its advantages, 2D model-based positioning technologies are widely used around the world. In contrast, 3D model-based positioning technologies mostly use TOA (Time of Arrival method), AOA (Angle of Arrival method) and other positioning methods based on time and space attributes. These types of technologies require an additional hardware platform, which greatly increases costs, but positioning accuracy is higher. As the positioning results are maintained at a decimeter level, the technology also has considerable applications in specific fields. The fingerprint positioning technology based on Wi-Fi is one of the most popular positioning technologies based on 2D modeling, and has become an area of indoor location research as it can be used in both outdoor and indoor positioning. For outdoor positioning as per reference [11], outdoor fingerprint positioning technology mainly uses three types of fingerprint. The first type is a visual fingerprint like image; the second type is a motion fingerprint, which uses motion sensors such as accelerometers, electronic compasses, etc.; and the third type is a signal fingerprint like RSSI (Received Signal strength indication). In comparison, indoor fingerprint positioning technology mainly uses signal fingerprint and uses the received signal strength of every location (as fingerprints stored in the fingerprint database) to match the signal strength measured at users' locations to achieve positioning. This paper focuses on the fingerprint positioning technology based on $\mathrm{Wi}-\mathrm{Fi}$, as shown in Figure 1 (which is based on the Figure 1 in reference [12]).

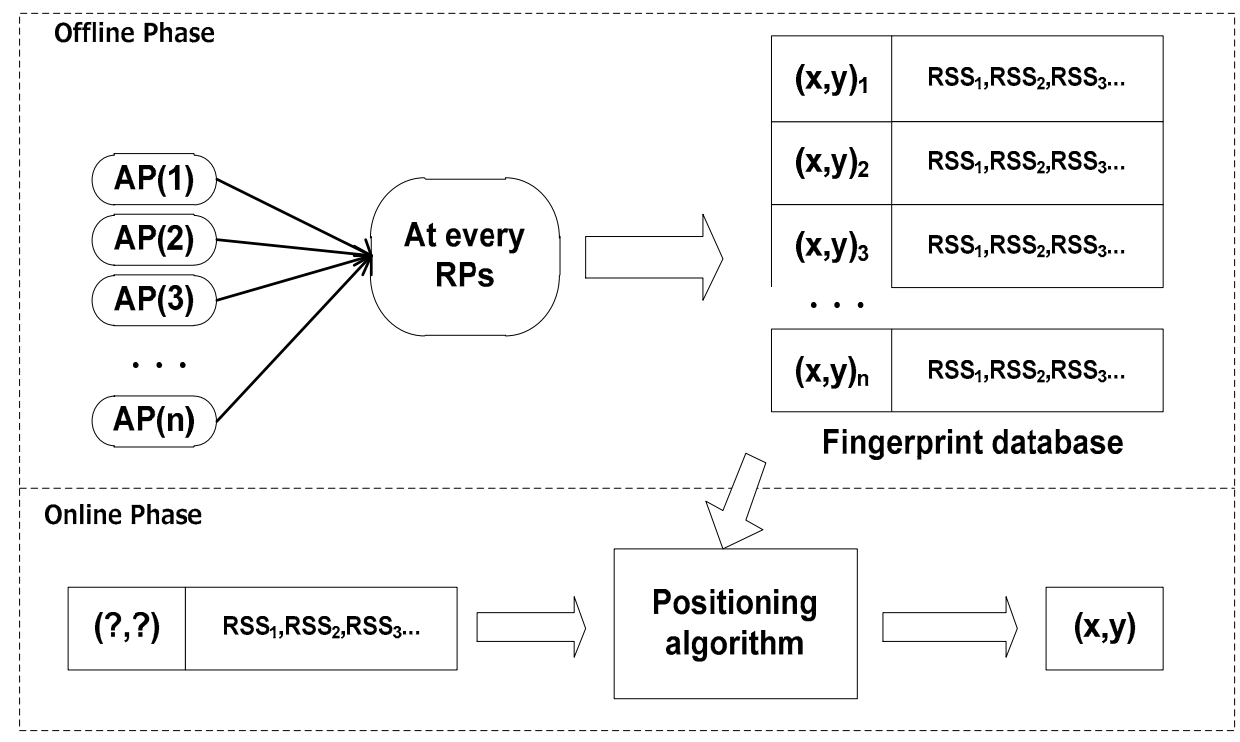

Figure 1. Technology of indoor fingerprint positioning based on Wi-Fi.

The rest of this paper is organized as follows. Section 2 makes a comprehensive introduction to the positioning technology based on Wi-Fi; Section 3 mainly focuses on fingerprint positioning technology based on Wi-Fi; Section 4 looks at auxiliary positioning technology with inertial sensors; Section 5 mainly introduces some public databases and competitions in this field; and Section 6 concludes the paper and addresses directions in future research. 


\section{Wi-Fi-Based Positioning Technology Theory}

\subsection{Wi-Fi Working Principle}

Wi-Fi (Wireless Fidelity) is a wireless local area network (WLAN) standard, which is a form of local area network connecting equipment used in a small space through a radio frequency signal. Not only can Wi-Fi identify the interconnection of the apparatus, but can also connect to the wider network through Wi-Fi.

$\mathrm{Wi}$-Fi is the IEEE $802.11 \mathrm{~b}$ standard, using radio frequency technology (RF) electromagnetic waves as the data carrier. Due to the high frequency $(2.4 \mathrm{GHz}$ and $5 \mathrm{GHz})$ and short transmission radius of the Wi-Fi signal (around $100 \mathrm{~m}$ ), it is difficult to cover whole large areas with Wi-Fi and can only be used in local hotspots such as apartments, offices, markets, etc.

\subsection{Wi-Fi Positioning Technology}

The market potential of indoor positioning technology has greatly improved with the wide distribution of Wi-Fi. The high precision, low-power consumption, and low-cost has made it an area of great interest in indoor positioning technology research. According to the IEEE 802.11 standards, a Wi-Fi wireless network card and access point (AP) has the function of measuring the intensity of radio frequency signals [13]. Therefore, users can utilize mobile devices such as smart phones, laptops, tablet PCs, and others to achieve indoor positioning with Wi-Fi and certain algorithms. So far, there are two types of Wi-Fi-based positioning technologies: time and space attributes of received signal (TSARS)-based technology and received-signal strength (RSS)-based positioning technology.

\subsubsection{Time and Space Attributes of Received Signal-Based Positioning Technology (TSARS)}

TSARS-based positioning technology mainly uses time and space attributes of received signal such as spatial distance, the time signal sending-receiving consumes and the spatial angle of signal. This technology includes TOA, AOA, and Time Difference of Arrival (TDOA) [14]. TOA calculates the distance according to the arrival time of the signal sent by different APs (Access Points) [15]. TDOA measures the delay time of the arrival signal [16], while AOA measures the angle of the arrival signal.

After obtaining the space-time relationship between the user and multiple APs, the user's position is calculated using trilateration [17]. To achieve high accuracy, at least three APs are required in this method. In some cases, additional hardware devices are needed, which leads to high deployment cost. As RF signal propagation in indoor environments is complex and can be affected by the multipath effect-human body interference and other factors-which creates many difficulties when measuring the RF signal and greatly affects positioning results.

\subsubsection{Received Signal Strength-Based Positioning Technology (RSS)}

The RSS-based positioning technology utilizes the strength of the received signal to obtain the users' position. This method does not require an additional positioning device. This method has attracted immense attention due to its high accuracy, low-cost, and low-power consumption since it was first developed. In general, there are three kinds of the method [18]: trilateration, similarity perception, and scene analysis, as shown in Figure 2. 


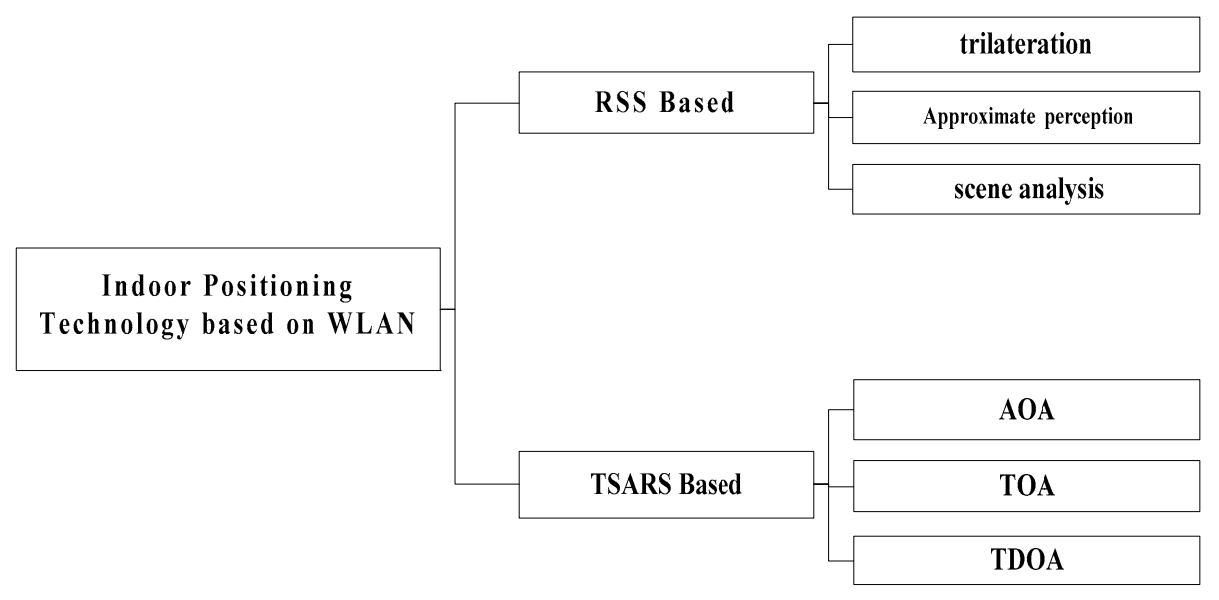

Figure 2. The classification of indoor positioning.

\section{(1) Trilateration}

Trilateration is a typical form of positioning technology which has been extensively studied and applied in the process of indoor positioning technology development. Researchers combined a triangular positioning method with the TSARS positioning technology, which has also reached a certain effect. Due to the limitations of the above-mentioned techniques and the advantages of signal strength (RSS), the triangular positioning technology based on RSS has been widely studied.

The trilateration method uses three or more APs to send signals received by the mobile devices and converts them into spatial distance which is used as the radius of circles (whose center is AP) and the intersection of the three circles is the measured user location, as shown in Figure 3.

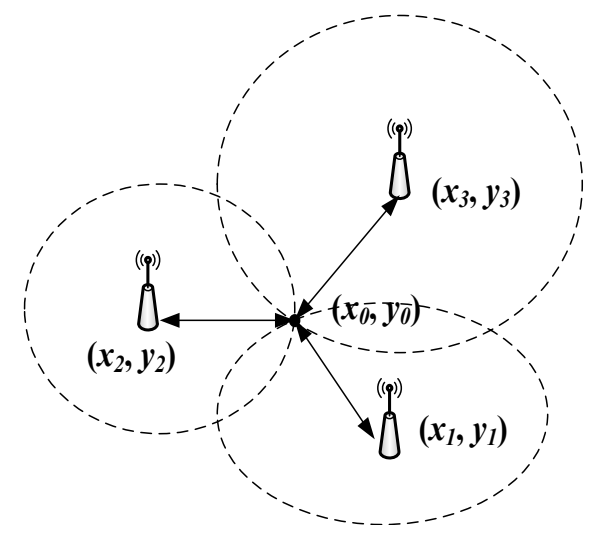

Figure 3. Trilateration.

Since the complexity of indoor space can have a great impact on the RF signal, when one converts the signal strength to spatial distance, it can inevitably produce errors. To reduce the error, researchers have suggested a variety of methods to assist trilateration. Among them, the path loss model to estimate the space position of APs is the most widely used.

There are two kinds of indoor path loss model [19]: the empirical path loss model, and the classic logarithmic model. Based on these two types, researchers have greatly improved the accuracy of trilateration in combination with other methods. In 2013, Bai et al. [20] proposed a method combined with the KNN (K-Nearest Neighbor) algorithm and fingerprint positioning technology; the triangular positioning method was based on the classic path loss model where they found that the positioning accuracy of their method increased $0.5 \mathrm{~m}$ compared with the Ekahau real-time positioning system [20]. The Active Campus system Griswold et al. [21] proposed in 2004, used the classic path loss model 
combined with the trilateration positioning method for indoor positioning where the positioning accuracy of it remained in the 8-10 $\mathrm{m}$ range.

Due to the need to obtain precise AP positions and the use of the accurate path loss model, the triangular positioning method is less practical, difficult to locate, and has low positioning accuracy.

(2) Approximate Perception

The approximation perception (AP) method is a relatively simple method [22], which uses the strongest base station as the positioning criterion. When the user communicates with a wireless device via Wi-Fi, the AP (which the device is connected to) will be viewed as user position. Since this method depends heavily on AP positions and has low positioning accuracy, researchers used an antenna cluster at a known position to improve positioning accuracy [18].

In addition to the antenna cluster, Cell-ID-which is generally used in the cellular mobile network - can be used to locate by enhancing the spatial property and strength of a Wi-Fi signal. Cell-ID divides indoor space into cells with a unique ID through signal strength received from the base station.

Approximate perception is simple, but the method heavily relies on the base station location and coverage areas. In addition, this method has a low accuracy of around $100 \mathrm{~m}$.

\section{(3) Scene Analysis}

Scene analysis is also known as fingerprint matching. It differs from other methods as it does not require the location of the AP in advance, or the specific distribution of interior space environment or scene, just several APs and their corresponding algorithms can obtain precise location results. As scene analysis has the characteristics of being low-cost, high-precision, and low-energy consuming, this method has become of great interest.

Scene analysis is based on signal strength and the signal intensity of different APs at the same Reference Point (RP) is measured to obtain the strength information of the RPs. Combined with specific coordinate information and the MAC (Media Access Control) address of the RP, we can acquire data on the RP, also called a fingerprint. After all fingerprints are collected, researchers can use these fingerprints to establish a fingerprint database called the radio map. With the radio map, researchers can collect the user's signal strength and compare it using the fingerprint database stored in it via the corresponding algorithm to obtain the location of the user.

Scene analysis generally is divided into two phases. The first phase is the offline acquisition phase where the main task is to establish a fingerprint database. The second phase is called the online phase. In this phase, researchers utilize real-time receiving signal strength measured by mobile devices and find a fingerprint which mostly matches the measurements through specific matching algorithms in the database to obtain the relevant position of the user.

\subsection{Wi-Fi Positioning Metrics}

With the development of Wi-Fi positioning technology, many Wi-Fi positioning systems have been proposed-from early systems such as RADAR [2] and Horus [3], to systems using advanced technology such as WILL [23] and Lifts [24]. These systems, including the various techniques and methods researchers have proposed, all view accuracy as the primary measuring rule of indoor positioning technology. However, it is not enough to measure how well an indoor positioning technology is operating by only using accuracy to judge. According to reference [18], the Wi-Fi positioning system has the following several aspects of metrics.

\section{(1) Accuracy/Measurement Uncertainty}

Accuracy, or measurement uncertainty, is the primary measure of the indoor positioning method. The higher the accuracy, the better the positioning effect of the method, thus the greater the likelihood of the method being more suitable for indoor positioning. According to Mautz [25], instead of accuracy, 
measurement uncertainty is now used for the quantification of a standard deviation. However, many researchers still use accuracy in their studies so as to avoid misunderstanding, we use the conventional term 'accuracy' in this paper. Mautz [25] thought that "positioning accuracy" should be understood as the degree of conformance of an estimated or measured position at a given time to the true value, expressed for the vertical and horizontal components at the $95 \%$ confidence level. Of course, due to various application environments, each indoor positioning system has different requirements for accuracy.

(2) Precision

Precision is used to describe the continuous measurement performance of an indoor positioning method, and it is a standard used to judge the robustness of the indoor positioning method. Liu [18] proposed that precision should be used as the distribution of distance error between the estimated location and the true location.

Here, we introduce the cumulative distribution function to measure precision. When two indoor positioning methods are compared, if the accuracy of both are the same, the faster the cumulative distribution function curve grows, and the faster the probability of reaching the peak, the better the method's performance. As the error distance interval considered by the cumulative distribution function of precision is very small, researchers generally use percentages to value precision. For example, if the precision of one indoor positioning method is $90 \%$ within $2 \mathrm{~m}$, this means the cumulative distribution function of the error distance is less than 0.9 within $2 \mathrm{~m}$.

\section{(3) Complexity}

Complexity is an important criterion to measure an indoor positioning method. Low complexity methods have better adaptability to the dynamic change of the indoor space and the user's position transformation response. The complexity of an indoor positioning method is mainly composed of hardware distribution, algorithm, and an operator.

For hardware distribution, the more the hardware is distributed, the higher the costs, likewise, the more complex the positioning methods, the higher the complexity.

For the algorithm, the spatial complexity and time complexity of the algorithm used in an indoor positioning method can directly affect the performance of the indoor positioning method. Due to the limited processing capacity of wireless terminal equipment, especially mobile devices, the low spatial complexity of a positioning method represents low energy and memory consumption, while low time complexity represents low positioning delay and high dynamic adaptability.

With regards to the operator, an indoor positioning method that introduces fewer operators means less preliminary work which means that less information is required. This makes for a better user experience.

\section{(4) Robustness}

Robustness describes the robustness and adaptability of indoor positioning methods. A highly robust indoor positioning method ensures high accuracy and precision even on the condition that errors occur in signal strength or radio map. Highly robust indoor positioning methods can be applied to a variety of indoor environments, whether it be free space or a harsh indoor environment.

\section{(5) Scalability}

Scalability describes whether the positioning method can maintain good performance when the environment expands. In indoor space, the farther the distance between the user and APs, the lower the performance of the positioning method. An indoor positioning method considers two aspects of the interior space: geographical distribution and density. Geographical distribution characterizes the spatial structure of the interior space. The more complex the geographical distribution of interior space, the greater the impact on the signal. Density represents the number of units of geospatial location per unit time. With the increase in density, geographic space becomes larger, which requires better 
scalability. Another aspect of scalability is dimensionality as some positioning methods are applied in two-dimensional space, and others are applicable in three-dimensional space.

(6) Costs

For an indoor positioning system, the costs encompass several aspects, including capital expenditure, time consumption, space consumption, energy consumption, and sink consumption. The cost of capital is crucial to the usefulness of an indoor positioning method, mainly due to hardware costs. Time cost includes deployment and maintenance time. Space consumption mainly refers to the unit density of the method. Due to the limited energy of the mobile terminal, energy consumption is particularly important. The sinking cost refers to whether the hardware devices used in the deployment of an indoor positioning method already exist or not. In comparison, low-cost indoor positioning methods are likely to be more popular.

Furthermore, Mautz et al. [25] discussed more metrics such as coverage area (which describes the spatial extension where system performance must be guaranteed by a positioning system), continuity (which is the property of continuous operation of the system over a connected period of time to perform a specific function), and so on.

\section{Fingerprint Positioning Based on Wi-Fi}

\subsection{Wi-Fi Fingerprint Positioning Technology Analysis}

To date, most of the indoor positioning methods are based on fingerprint matching technology combined with other methods [26]. With further study, researchers have used many ways to improve fingerprint matching technology across all aspects.

\subsubsection{Advantages of Wi-Fi Fingerprint Positioning Technology}

Compared with other positioning systems, Wi-Fi fingerprint positioning technology has the advantages of low-cost and high precision. Due to the wide deployment and use of Wi-Fi worldwide, fingerprint positioning technology can be applied to any indoor scenario where Wi-Fi networks are deployed without any additional hardware, which makes the technology cost low. This technology uses Wi-Fi signal strength to model and measure, without having to identify the exact location of the APs. In a complex indoor environment, under low-cost conditions, the space-time attributes such as angle and time of arrival can produce large errors; however, the signal intensity is relatively stable, thus making the positioning accuracy of this method greater than others.

\subsubsection{Disadvantages of Wi-Fi Fingerprint Positioning Technology}

Wi-Fi fingerprint positioning technology needs many algorithms as support, so computation consumption and algorithm complexity is relatively high. Additionally, as the method requires a large amount of a priori information as data support, the preliminary work has a high cost factor. Since the method is modeled by the strength of each AP signal received at a certain location, it is also influenced by the environment. Once the AP location or the indoor environment changes, data re-acquisition and re-modeling are needed. The indoor environment is complex, so radio frequency signals will be affected by multipath effects, so there may be errors in the measured signal intensity.

\subsubsection{The Influence Factors of Wi-Fi Fingerprint Positioning}

(1) Effects of the Human Body on Signal Strength

As $70 \%$ of the human body is composed of water, it can partly absorb the radio frequency signal of $2.4 \mathrm{GHz}$ [27]. Since people spend most of their daily time in an indoor space, the human body has a significant impact on radio frequency signal by causing the decline and uncertainty of the signal in varying degrees. 
Walters [28] suggested that the location of the human body in an indoor space, the distance between people and APs and human body orientation could influence signal propagation. Walters found in their experiments that when there were moving bodies in indoor space, the average signal strength declined and the standard deviation increased significantly. Additionally, when mobile devices were completely blocked by human bodies, the signal strength would decrease significantly by around $10 \mathrm{dBm}$.

(2) Effects of the Multipath Effect on Signal Strength

Since many obstacles (like windows, doors, and walls) exist in indoor space, the radio frequency signal will propagate through different paths, which causes the signal to reach the receiver at different times. A different receiving time causes different phase, which can be superposed and cause signal distortion. This phenomenon above is called the multipath effect.

Multipath is the main factor affecting indoor signal strength and the multipath effect is different in diverse environments due to their different distribution, construction, main materials [29], and so on. To minimize its impact, researchers have used the channel propagation model. Fang et al. [30] found that the multipath effect could be divided into two parts: one part is the convolution filter effect caused by multi-scattering in an indoor environment, and the other part is communication additive noise. They modeled both effects with the logarithmic propagation model and reduced the probability of the standard deviation of estimate error from $42 \%$ to $29 \%$, respectively.

It is worth mentioning that, due to the multipath effect, there is time-duration exiting while measuring the RSS, which also has an impact on RSS measurement. Ignoring the time-duration leads to incorrect and unstable RSS measurements. Thus, it is necessary to keep measuring for a period, like 20 to $30 \mathrm{~s}$, depending on the indoor environment [31].

(3) Effects of the Number of APs and RPs

The number of APs and RPs has a profound effect on the fingerprint positioning methods. Too few results in inaccurate fingerprint data, leading to poor performance. However, blindly increasing the number can cause further costs and an extension of time during the offline phase. According to different indoor environments, selecting the appropriate number of APs and RPs is a mean in which to improve the efficiency of indoor positioning.

Moghtadaiee [32] analyzed and modeled the number of APs and RPs. In their experiment, they used the root mean square (RMS) deviation of various positioning algorithms (like KNN and ANN) and the lower bounds of the non-Bayesian probability method with different numbers of APs and RPs and found that in an indoor space with dimensions of $11 \times 23 \mathrm{~m}$, the RMS of different methods can be as low as 5 APs and increasing their number barely influences the result. Similarly, the RMS of different methods hardly changes with 66 RPs and more RPs are useless.

\section{(4) Effects of Mobile Devices}

Walters [28] suggested that the RSS (Received Signal Strength) measured at the same location by dissimilar devices could be different. The real received signal energy is measured in $\mathrm{dBm}$ or decibel milliwatt, but researchers generally use RSS measured in $\mathrm{dBm}$ as an integer number [28]. Different device manufacturers have different standards to convert the real signal energy strength to an RSSI value, which causes differences in RSS measurement that can be large in some cases.

In addition to the type of mobile devices, their orientation also has a large impact on the RSS value. Kaemarungsi and Krishnamurthy [31] discussed the orientation of mobile devices in their work and found that the RSS values measured by devices oriented in various directions at the same location could be different. They further found that if the device was set behind the APs, the RSS value was the lowest, which decreased by $10 \mathrm{dBm}$ when compared with the highest which was measured when the device was set facing the APs. This indicated that device orientation is crucial and should be considered during RSS measurement. 


\subsection{Fingerprint Data Collection}

Fingerprint data collection, also called offline training, is the first phase of fingerprint positioning, which uses the fingerprinting technique to store the location dependent characteristics of a signal collected at known locations ahead of the system's use for localization in a database [33]. The main task in this phase is to collect fingerprint data to build a model. There are two main methods of collecting fingerprint data, one is accurate modeling and the other is empirical modeling [34].

\subsubsection{Empirical Modeling}

Empirical modeling is determining the number of RPs at certain intervals in the area to be located, and measuring the signal strength received from different APs at the location of every RP. The signal strength is stored with information of the RP's location, as well as other information (like MAC address) in the fingerprint database or radio map as a piece of fingerprint data. Since empirical modeling is based on many data samples, the workload in the early stages can be large and this modeling method depends on the indoor environment. However, this modeling method has a high level of accuracy.

According to Jung [35], construction of the empirical models involves fingerprint data collection and the fingerprint data component. Fingerprint data collection is how fingerprint data samples like RSS - which are used to build the empirical models or radio maps-are obtained. The fingerprint data component is another important factor as different positioning strategies use different data based on RSS.

There are different strategies to collect fingerprint data. To cover the entire area, researchers distribute different numbers of AP and select various numbers of RP based on indoor environments. The most primitive way is by point-by-point manual calibration, which can achieve the greatest accuracy. The target area is partitioned into numerous pieces, i.e., locations, and dedicated surveyors collect fingerprint samples point-by-point by considering the center of each location as a measurement point [35].

Liu et al. [36] proposed a strategy using the smallest enclosing circle algorithm (SEC) which is widely used in the field of information security. They directly input the RP's coordinates instead of the RSS, and the smallest enclosing circle is the smallest circle that consists of all points to be positioned. Associated with the WKNN (Weighted K-nearest Neighbors) algorithm and clustering, this method decreased the calibration cost and improved the positioning accuracy by $29.1 \%$.

Fetzer et al. [37] proposed a strategy that combined varieties of assistive technology aimed at multi-floor indoor environments. The fingerprint data they used is described in Equation (1).

$$
o=\left(s_{\text {wifi }}, s_{i b}, \Delta \theta, n_{\text {steps }}, \rho_{\text {rel }}, \Omega\right)
$$

In Equation (1), $S_{\text {wifi }}$ and $S_{i b}$ contain the RSS measured from the AP and i-Beacons, respectively. $\Delta \theta$ and $n_{\text {steps }}$ describes the relative angular change and the number of steps detected for the pedestrian. $P_{r e l}$ indicates the relative barometric pressure used to detect the floor where users were located. Finally, $\Omega$ contains the activity currently estimated for the pedestrian by accelerometer and are: unknown, standing, walking, walking up the stairs, or walking down the stairs.

To obtain the fingerprint data, they arranged four different walking paths instead of point-by-point manual calibration. This method is called a walking survey [35] and reduces the effort required to recognize each location. Moreover, this method was also used to obtain sensor signals like barometric pressure and the activity of the pedestrian. However, walking surveys require additional time, which increases the cost of effort.

The IPIN (Indoor Positioning and Indoor Navigation) competition discussed in [38] introduced several new indoor positioning systems. The team called HFTS used the walking survey method to acquire fingerprint data containing heading directions, RSSI and step information, while the UMinho team's approach was based on Wi-Fi combined with placement records and assistive positioning technologies. The team tried different strategies to build the radio map, which was finally built by 
associating fingerprints to linearly interpolated coordinates between known positions, combined with the movement information extracted from the accelerometer data. Although many other strategies were introduced in reference [38], they will not be discussed in this paper.

Another indoor positioning competition called the EvAAL-ETRI Indoor Localization Competition (part of the Sixth International Conference on Indoor Positioning and Indoor Navigation (IPIN 2015) was presented in reference [39] where many novel and effective approaches were introduced. Additionally, references [40-42] discussed different ways to build empirical models by using multi-points, crowdsourcing, and so on.

\subsubsection{Accurate Modeling}

Accurate modeling—also called channel propagation model modeling—only needs the signal strength of several important positions to generate the entire radio map by calculating and expanding the data with the channel propagation model. Accurate modeling requires the accurate position of the APs; however, the position can be hard to determine in most cases. Thus, channel propagation modeling can be used in an experimental environment where the position of the AP is known to find the relationships between fingerprint data [33], or examine the performance of the proposed method. Furthermore, this modeling can be used in a real positioning environment to calibrate the positioning results obtained by other means.

For radio frequency signals, signal propagation is always affected by scattering, reflection, and refraction. Due to the complexity and limitations of the indoor environment, this effect can be amplified, making the signal transmitted from the broadcaster to the receiver over a variety of paths. Called the multipath effect, it causes signal fading and phase shift [2], which decreases the signal-to-noise ratio. Inaccurate signals can reduce the accuracy of positioning and bring larger distance errors. Therefore, the main task of accurate modeling is to establish a channel propagation model which can describe indoor signal fading properly.

\section{(1) Typical Channel Propagation Models}

Due to the complexity and limitation of the indoor environment and the large number of obstacles, there are four factors to be considered when building a channel propagation model: path decline, scattering, refraction, and reflection $[43,44]$. As such, the channel propagation model should be combined with a large-scale fading model and a small-scale fading model [45]. Here, we introduce several typical channel propagation models [34].

\section{Indoor General Propagation Model:}

$$
P L(d)=P L+10 \times N s f \times \log _{10}(d)+F A F
$$

In Equation (2), $P L$ represents the path loss in the range of $1 \mathrm{~m}$ in free space, while Nsf represents the dissipation factor of the same layer, and $F A F$ represents the additive value of the loss through different paths. The model does not take the obstacles in actual interior space into account, and one has to measure the actual field strength to acquire the value of Nsf and FAF before using this model.

Chan Propagation Model:

$$
L=P L_{0}+10 \times n \times \log _{10}(d)+F_{\text {wall }}+F_{\text {floor }}
$$

In Equation (3), $L_{f_{s}}$ represents the pass loss in free space and $L_{p}$ represents the loss value associated with the interior walls. The Chan propagation model is the most basic prediction model of indoor path loss, and it takes the impact of indoor walls on channel propagation into account based on the free space propagation model.

Loss Factor Propagation Model:

$$
L(\mathrm{~d})=P L+10 \times \eta_{s f} \times \log _{10}(d)+F A F
$$


In Equation (4), $P L$ represents the path loss in the range of $1 \mathrm{~m}$ in free space; $\eta_{s f}$ represents the testing index of area to be tested; and FAF represents the additive value of the loss through different paths.

\section{Motely Propagation Model:}

$$
L=P L_{0}+10 \times n \times \log _{10}(d)+F_{\text {wall }}+F_{\text {floor }}
$$

In Equation (5), $P L_{0}$ represents the path loss in the range of $1 \mathrm{~m}$ in free space, while $n$ represents loss factor (value recommended as 2 in this model). $d$ represents the distance between wireless devices; $F_{\text {wall }}$ represents the correction factor of the signal attenuation caused by walls; and $F_{\text {floor }}$ represents the correction factor of the signal attenuation caused by floor. Both correction factors change with materials.

\section{Application of Channel Propagation Model:}

The radar [2] system analyzed the ground attenuation factor channel model (FAF) [46], and found that when this model was associated with a large-scale path loss model, it was flexible enough to be used in different indoor space structures. Based on the FAF, researchers built a model called the wall attenuation factor channel model (WAF) used in radar, as shown in Equation (5).

$$
P(d)[d b m]=P\left(d_{0}\right)[d b m]-10 n \log _{10}\left(\frac{d}{d_{0}}\right)- \begin{cases}n W \times W A F & n W<C \\ C \times W A F & n W \geq C\end{cases}
$$

In Equation (6), $n$ represents the distance path attenuation rate, where $P\left(d_{0}\right)$ represents the signal strength value under the condition that the distance between the sender and receiver equals reference distance $d_{0}$; and $d$ represents the distance between the sender and receiver. $C$ indicates the maximum number of walls that the factor works; $n W$ represents the number of walls between the sender and receiver; and WAF represents the wall attenuation factor. Using the WAF model, the median of accuracy of positioning can reach $4.3 \mathrm{~m}$.

Nurminen et al. [47] used the classical logarithmic model (power-law model) as the indoor channel propagation model. The classical logarithmic mode combines LOS (Line of Sight) and NLOS (Not Line of Sight) propagation by using numbers of correction factors to offset the uncertainty caused by these two propagations. Equation (7) shows the logarithmic model Nurminen [47] used.

$$
P_{r x}(d)=P_{r x}\left(d_{0}\right)-10 \times n \times \log _{10}\left(d / d_{0}\right)+W
$$

$P_{r x}(d)$ is shown as a logarithm, where $d_{0}$ represents the reference distance. $n$ represents the path fading index and $W-N\left(0, \sigma^{2}\right)$ represents a random variable as normal distribution, which indicates the slow fading (shadow fading) of indoor signal propagation. Nurminen [47] utilized this model based on a large number of fingerprint data measured to optimize his research results, which had some effects.

Laitinen et al. [48] improved the model proposed by Nurminen [47] and built the single slope model, shown in Equation (8).

$$
P_{i, a p}=P_{\text {Tap }}-10 n \log _{10} d_{i, a p}+\eta_{i, a p}
$$

In Equation (8), $n_{a p}$ represents the effect of path loss on the ap-th AP where $d_{i, a p}$ represents the distance between the ap-th AP and the i-thRP, which is calculated as Euclidean distance. $P_{i, a p}$ represents the signal strength measured at the i-thRP sent from the ap-th AP, where $P_{\text {Tap }}$ represents the signal propagation strength of the ap-th $\mathrm{AP}$, and $\eta_{i, a p}$ represents a noise factor as normal distribution. Unlike Nurminen [47], Laitinen [48] obtained only a few pieces of fingerprint data at several key RPs and then obtained the entire radio map through the model. Laitinen [48] optimized the fingerprint database in this way, which improved positioning efficiency. 
Alternatively, there are other new ways to achieve positioning using accurate modeling in combination with other methods like those discussed in reference [49].

\subsection{Signal Pre-Processing}

The main purpose of signal pre-processing is to optimize the fingerprint database and remove invalid and redundant data by filtering and clustering, to improve the operating efficiency of the whole positioning system.

\subsubsection{Fingerprint Database Denoising}

During the fingerprint acquisition process, the complex indoor environment can have a great impact on the signal strength by generating a large number of noise, which is very difficult to eliminate regardless of the selected acquisition method. Therefore, it is necessary to filter the fingerprint database, not only to optimize the sample space and remove the invalid samples and bad data, but also to improve positioning accuracy and enhance the efficiency of the positioning system. So far, based on the differences between all kinds of positioning algorithms, there are two kinds of methods in fingerprint filtering: deterministic denoising and probabilistic denoising.

\section{(1) Deterministic Denoising}

Channel Propagation Model Denoising: More than $90 \%$ of indoor channel propagation models are empirical models. The model is based on a large number of prior data, which has a high reliability. The channel propagation model is used to model the indoor space, and a correction factor is introduced to eliminate the influence of indoor areas (such as walls, doors, and windows) on the radio frequency signal, so the denoising of the channel propagation model performs well. The channel propagation model denoising uses fingerprint data as a parameter to be calculated in the channel propagation model after the end of the offline phase. The channel propagation model identifies the effective and invalid data, thus removing the noise. Channel propagation model denoising is a commonly used deterministic denoising method; however, this method mainly relies on the establishment of the channel propagation model, so the channel propagation model directly affects the denoising effect.

The radar [2] system used the channel propagation model to establish the fingerprint database, and at the same time, the radar system did the denoising operation to the fingerprint data.

Median Filter: Median filtering is a non-linear filtering denoising method that uses the median value of the neighborhood noise point to replace it. Lim et al. [50] chose median filtering to denoise the fingerprint database. The median filtering can effectively solve the short-term effect of fast fading on signal strength.

Neighborhood Mean Filtering: Like median filtering, neighborhood mean filtering is also a common denoising method. After finding the noise point of the fingerprint database, the value is estimated by using the average of the values of non-noise point in the neighborhood to achieve filtering [13].

In reference [13], after the neighborhood filtering method was used to denoise the fingerprint database, the average location error decreased by $13 \%$, and the error in the $6 \mathrm{~m}$ above the fixation point decreased by $46 \%$.

\section{(2) Probabilistic Denoising}

Probabilistic denoising is combined with probabilistic positioning algorithms. After establishing the fingerprint database in the offline phase, unusual data in the fingerprint database can be removed by establishing the probability distribution function. According to the probability distribution function, the most common method is the histogram method [34]. 


\subsubsection{Fingerprint Database Clustering}

With the indoor environment continually expanding, the required fingerprint data gradually increases. This increase directly affects the positioning efficiency and time consuming, which can also increase the number of invalid fingerprint data, resulting in reducing positioning accuracy, so the optimization of fingerprint data is particularly necessary.

Clustering evaluates the fingerprint data based on their similarity degree and classifies them by it to optimize the fingerprint data. After clustering, the fingerprint database can be divided into several parts, improving the efficiency of positioning and reducing the system consumption. Swangmuang [34] used median clustering and K-mean clustering to cluster the fingerprint database separately. The contrast experiment they undertook indicated that the clustering method could save many operations required from the model without clustering, which reduced around a third of the operations separately. Altintas [1] proposed a positioning solution using K-means clustering in combination with the KNN method which outperformed the traditional KNN. Instead of clustering the fingerprint database, they clustered the $k$ reference fingerprint data obtained by KNN with K-means clustering to participate in positioning. The K-means clustering reduces the required reference data and decreases the positioning average distance errors.

Lee [51] proposed a novel clustering-based approach to achieve indoor positioning. They presented the support vector machine-based clustering approach, named SVM-C, which used the margin between two canonical hyper planes for classification instead of using the Euclidean distance between two centroids of reference locations. They clustered the fingerprint data with SVM margins in the offline phase and utilized clustering match technology in the online phase. The experimental results indicated that the positioning mean errors were lower than other approaches like the means clustering method, SVC, etc.

\subsubsection{AP Selection Strategy}

With the constant development of $\mathrm{Wi}-\mathrm{Fi}$, AP distribution density is also rapidly increasing. Traditional indoor positioning methods can choose to use all the APs that can be detected in the environment; however, the signal strength provided by the AP can be affected by a severe multipath effect and has a low reference value which interferes with the accuracy of the fingerprint data and increases the burden of the positioning system, thus making it difficult to locate the fingerprint. Therefore, the choice of AP becomes an issue requiring further attention [52].

Jhuang et al. [53] proposed that, in the online phase, the standard error of the signal strength was used as the metric to calculate the detected signal strength of each AP and categorized them prior to selecting the top M APs as positioning APs to participate in positioning.

Feng Chen [54] listed two common AP selection strategies based on the strongest received signal strength and the Fisher criterion.

During the online phase, the AP selection strategy based on the strongest received signal strength chooses the $M$ strongest signals of the AP to participate in positioning. As this method sorts all the APs based on the strength of signals every time positioning, every time positioning it picks different APs to participate in positioning based on the different locations of users.

In contrast, the Fisher criterion calculates the signal strength from each AP through the fingerprint database analysis of signal distribution, as shown in Equation (9).

$$
\xi_{i}=\frac{\sum_{(j, o) \in C}\left(\varphi_{i, j}^{o}-\bar{\varphi}_{i}\right)^{2}}{\sum_{(j, o) \in C}\left(\Delta_{i, j}^{o}\right)}
$$

Among them, $\bar{\varphi}_{i}=1 / \tilde{N} \times \Sigma_{(j, 0) \in C} \times \varphi^{\circ}{ }_{i, j}$. As seen in Equation (9), the smaller the denominator, the higher the probability that the fingerprint data from the same AP is similar to the signal strength obtained at the same location in the online phase. Molecules represent the changes in signal strength 
at different RPs to measure the ability of different APs to differentiate reference points. The Fisher criterion selects the top $\mathrm{M} \xi_{i}$ of the largest Aps to participate in positioning by sorting $\xi_{i}$.

\subsection{Positioning Algorithm}

In the online phase, positioning systems match pre-collected data with the signal strength at the user's location to determine where the user is, and during this phase the use of positioning algorithms is important. So far, there are two kinds of positioning algorithms, one is the deterministic positioning algorithm and the other one is the probabilistic positioning algorithm.

\subsubsection{Deterministic Positioning Algorithm}

The deterministic positioning algorithm utilizes real-time matching of fingerprint data by means like machine learning, data mining, artificial neural network, etc. This method is based on the measurement of signal strength; therefore, it is affected greatly by the precision of the fingerprint data acquired offline.

(1) K-Nearest Neighbor

The KNN algorithm is one of the simplest algorithms in machine learning. KNN and its variants have been widely used in indoor positioning for its low-cost and high performance. The core thought of $\mathrm{KNN}$ is to compare the signal strength obtained by users with fingerprint data in the fingerprint database while positioning. It chooses the $\mathrm{k}$ nearest neighbors of fingerprint data according to root-mean-square error. It completes the positioning operation by calculating the weighted average of the $\mathrm{k}$ fingerprint data. The Weighted K-Nearest Neighbor is a variant of KNN that adds distance as weight. Nearest Neighbor is another variant of KNN.

The signal intensity vector of the user's location is $V_{a}=\left(V_{1}, V_{2}, \ldots, V_{n}\right)$ and is used to calculate the similarity between the signal intensity vector of the user's location and the fingerprint data in the fingerprint database. The computing method is shown as Equation (10).

$$
d_{q}\left(V_{a}, V_{i}\right)=\left(\sum_{j=1}^{n}\left|v_{j}-v_{i j}\right|^{q}\right)^{\frac{1}{q}}
$$

If it is calculated by the Manhattan distance, then $q=1$. If it is calculated by Euclidean distance, then $q=2$. After calculating the similarity, this method chooses the $k$ fingerprint data based on the similarity. The $k$ fingerprint data which contains the coordinates of the $k$ position is the most likely position of users and calculating the mean values of the coordinates of the $k$ fingerprint data increases accuracy. The average value is the location of the user.

The radar [2] system is the earliest indoor positioning system that applies the nearest neighbor methods. Bahl et al. presented a new version called the Nearest Neighbor in Signal Space by improving the nearest neighbor methods. The core ideal of the NNSS was to match the nearest neighbor by calculating the distance between the signal strength received by users and the signal strength in the fingerprint database. Bahl [2] compared this method with the perceived similarity and channel model methods and found that the positioning accuracy of this method could reach $2.94 \mathrm{~m}$. It still has some issues in its practical applications, but has provided new thoughts in researching indoor positioning.

Ma et al. [55] presented a new method called the Clustering Filtered KNN (CFK) which combined clustering with the KNN method. CFK can divide the entire fingerprint database into several non-adjacent parts by using hierarchical clustering as per the physical location of each neighbor point. It selects the nearest neighbor points in one cluster to match the user's location. Ma [55] also found that the average positioning error and positioning median error were both less than the KNN, as was the calculating cost.

Guowei et al. [56] used the Jeffries Matusita distance instead of the Euclidean distance. The distance formula was established based on Euclidean distance, but expanded the error function 
elements (which were small), and could stably achieve the Euclidean distance. First, their method obtained the nearest neighbor point datasets by KNN before extracting the reference points from these sets by the K-n algorithm. Finally, they used similarity between the user and reference points, and set the frequency of the reference points (showing as weight) to participate in matching. Compared to $\mathrm{KNN}$, the method put forward by Guowei [56] has carried out deeper digging on the correlation of signal strength value and has some advantages.

$$
d(x, y)=\left[\sum_{i=1}^{N}\left(\sqrt{x_{i}}-\sqrt{y_{i}}\right)^{2}\right]^{\frac{1}{2}}
$$

Shin et al. [57] presented the EWKNN (Enhanced Weighted K-nearest Neighbor algorithm) algorithm. First, it generated a list of signal strength by comparing fingerprint data in the fingerprint database with signal strength measured while positioning for the first selection. Next, it compared the rest of the data in the list for the second selection. The $k$ in Equation (11) represents the number of RPs reserved and utilizes the differences in signal strength as weight to participate in the second selection. To acquire the final positioning result, it requires some iterations. The EWKNN reduces the error in comparison to the $\mathrm{KNN}$ algorithm, and has better adaptability for $k$ which is variable.

\section{(2) Support Vector Machine}

Statistical learning theory is an important theoretical basis for the machine learning method, the theory of statistical estimation, and the prediction of small sample theory. The support vector machine (SVM) is one of the youngest, most practical, and highest potential methods in statistical learning theory as it translates the input space into a higher dimensional space by nonlinear transform defined by inner product function, and calculates the optimal classification plane in this space. The expression formula is shown by Equation (12).

$$
f(x)=\operatorname{sgn}\left(\sum_{i=1}^{n} T_{i} y_{i} K\left(x_{i}, x\right)+b\right)
$$

Here, $T_{i}$ is the Lagrange multiplier corresponding with each sample. $b$ is the classification threshold and $K\left(x_{i}, x\right)$ is the optimal classification plane of the inner product function. This function can achieve linear classification after a nonlinear transformation and is the kernel function of support vector machines (SVM) which are classified by its kernel function. So far, the SVM used in indoor positioning generally divides the fingerprint matching problem into two kinds: the SVC problems and the Support Vector Regression (SVR) problems.

Brunato et al. [58] systematically illustrated the application background and principle of statistical learning theory and SVM applied in indoor fingerprint positioning, and established the theoretical foundation for the application of SVM in the field of indoor positioning. Brunato [58] used SVM to match fingerprint data and compared them with WKNN, Bayesian regression analysis and artificial neural network. They found that the error of positioning results by SVC was very low, and the results by SVR were roughly the same as the WKNN algorithm.

Abdou et al. [59] presented regression analysis positioning technology based on the similarity of support vector machines by combining SVM and cluster. The method clustered the fingerprints in the fingerprint database via the similarity propagation model in an offline collection phase, and studied each type of fingerprint data by SVM for correlation information based on the RPs and signal strength values. In the online positioning stage, the user's mobile device uploaded the collected signal strength information to the server. The server determined the user's signal strength information membership category through pattern recognition and used the strongest AP to choose the corresponding AP information. Next, the information would be served as the input of the support vector machine regression analysis to conduct positioning precisely. The positioning results were good, and positioning errors were far less than other methods such as KNN, and Bayesian. 
Wu et al. [60] combined SVM and particle filter. They classified the data in the fingerprint database as per the known position by SVM and used Pedestrian Dead Reckoning to achieve a positioning match, and optimized the data by particle filter. The positioning accuracy was $1.2 \mathrm{~m}$.

\section{(3) Artificial Neural Network}

As the principle of fingerprint data matching is similar to pattern recognition, a large number of artificial intelligence technologies used in pattern recognition can also be used in fingerprint positioning. Artificial neural network is one of the most important methods in machine learning. To date, most researchers use artificial neural network methods, such as multilayer perceptron, back propagation neural networks, multi-layer neural networks, and regression neural networks.

Dai et al. [61] introduced the Multi-Layer Neural Network (MLNN) into indoor positioning. MLNN is a forward artificial neural network with some hidden layers existing between its input and output. The MLNN designed in reference [61] consisted of three parts: the transmission element, denoising element, and positioning element. The MLNN can be directly calculated from the user's location without matching fingerprint data. The experiments showed that the average error of positioning by this method was less than other mainstream methods.

Fang et al. [62] proposed Discriminant-Adaptive Neural Networks. This method first uses multiple discriminant analysis to find useful discriminative information in the fingerprint data, and then introduces the extracted information into the discriminative components to increase the important information weights, maximizes the useful information in the neural network, and uses the information as the parameter to participate in the DANN (Discriminant-Adaptive Neural Network) calculation. Compared with other mainstream methods, this method achieved higher positioning accuracy.

Battiti et al. [63] used a multi-layer perceptron for indoor positioning, with a positioning accuracy of $2.3 \mathrm{~m}$. Nowicki et al. [64] proposed a method that used Deep Neural Network (DNN) combined with stacked autoencoder (SAE). The DNN used in this method was the first to be used for Wi-Fi fingerprinting and could achieve better performance in image analysis than other methods. The SAE Nowicki [64] used could determine the floor or building and reduce dimensionality of the input data. The experiment results were better than other networks without autoencoders.

(4) Other Deterministic Positioning Algorithms

In addition to the above-mentioned mainstream positioning algorithm, there are several algorithms used by researchers that have achieved good results. Kushki [65] used the distance calculation method of kernel function and AP selection strategy. The average positioning error could be kept at approximately $2 \mathrm{~m}$. Feng Chen [54] used the combination of compression perception technology and clustering technology for indoor positioning. Yim [66] used a decision tree for indoor positioning, which reduced not only the complexity of the algorithm, but also the average positioning error when the sample space was larger (about 80 samples), in comparison with other algorithms. $\mathrm{Li}[6]$ used the Least Square and its related variants, such as the Weighted Least Square (WLS), and the Nonlinear Least Square (NLS) to achieve positioning match. The average error could be up to $1 \mathrm{~m}$ and positioning efficiency was high.

\subsubsection{Probabilistic Positioning Algorithm}

The most significant difference between the probabilistic positioning algorithm and the deterministic positioning algorithm is that the deterministic positioning algorithm matches the signal strength of corresponding fingerprint data by using the positioning algorithm to obtain the final position of the user, while the probabilistic positioning algorithm stores the probability distribution of the signal strength during a certain time in the fingerprint database [22]. Next, the probability position of the user's location is calculated by the Bayesian theory system based on signal strength.

Kontkanen et al. [67] conducted a more comprehensive analysis and study of the application of probability and statistical methods in location services and concluded that the probability and 
statistical method not only had broad prospects in indoor positioning, but also had great potential in personnel tracking, distance calibration, and other related position services.

The main task of the probabilistic positioning algorithm in the offline phase is to build the probability distribution model of signal strength collected through the sample data by using corresponding methods such as the histogram method and kernel function. In the online phase, the model calculates the probability that a user is at a certain location, and then uses the model to estimate the location of the user.

(1) Probability Distribution of Signal Strength

An important step is to obtain the probability distribution of the signal strength for the probabilistic positioning algorithm. The quality of the probability distribution model directly affects the efficiency and accuracy of positioning. The probability distribution model of signal intensity is established using the histogram method or kernel function based on the signal intensity measurements of different APs received at different RPs in the offline phase.

Many researchers have used standard Gaussian distribution to model the probability distribution of signal strength. For example, Kaemarungsi et al. [31] analyzed the change and influence of signal intensity in the indoor environment and concluded that standard Gaussian distribution was more suitable for the probability distribution of the signal strength in the indoor environment. The Horus system, designed by Youssef et al. [3], was the first system to locate indoors through the probabilistic positioning method that also used a standard Gaussian distribution in the distribution model.

Luo et al. [68] suggested that the standard Gaussian distribution did not fully describe the signal strength in the indoor environment of the distribution. Luo [68] demonstrated that the signal intensity distribution in indoor environments tended to bimodal distribution rather than the traditional sense of the standard Gaussian distribution. They found that the signal strength values will be left, and its peak was much higher than the standard Gaussian distribution, so proposed the addition of appropriate parameters to perform a more suitable fit in the probability distribution of signal strength modeling. Chen Miao [69] proposed a multi-Gaussian mixture model to store the signal strength; and Ferris et al. [70] used a Gaussian process to model the signal strength. Although the Gaussian process was used to reduce the number of samples collected previously, the probability distribution model established at each reference point was the standard Gaussian distribution, which could not describe the signal strength in the indoor environment.

(2) Probabilistic Positioning

After establishing the probability distribution model of the signal strength, we needed the Bayesian system, combined with other methods (the most commonly used is clustering), on the posterior probability of the user's location calculated to obtain an estimate of the user's location. Naive Bayesian, Hidden-Bayesian, Bayesian networks, and maximum likelihood estimation are also widely used methods.

The Horus system uses joint clustering, probability distribution, and deterministic positioning, first combining the clustering and probability distribution in the indoor positioning field simultaneously. The Bayesian theorem and the maximum certainty positioning algorithm were used to solve the positioning problem in different environments. The positioning error of the system was less than $0.6 \mathrm{~m}$.

Bisio et al. [71] proposed an intelligent probabilistic fingerprinting algorithm that improved the computational speed of prior probabilities and reduced the cost of positioning and the time-consuming costs associated with traditional probabilistic fingerprinting algorithms. Madigan et al. [72] used a Bayesian hierarchical model for positioning. Patwari et al. [73] selected the maximum likelihood function for positioning. 


\subsection{Back-End Filtering}

Due to the complexity of the indoor environment and the wide distribution of noise, back-end filtering is needed to obtain more accurate results at the end of positioning. The commonly used filtering methods are Bayesian filtering, Kalman filtering, and particle filtering.

Honkavirta et al. [74] have described Bayesian filtering and Kalman filtering in detail. Gordon et al. [75] introduced the application of particle filter in location tracking. Wu Yao [76] also provided a more in-depth description of the fusion of the filtering algorithm and the location-tracking technique.

Back-end filtering has a certain effect on the improvement of positioning accuracy. However, it can affect the whole process of positioning if the cost of back-end filtering is too high, so most researchers choose mean-based filtering in the back-end filtering technology.

As most positioning results are relatively stable and accurate, and the cost of back-end filtering methods are relatively high, it is not widely used by positioning systems.

\section{Assistive Positioning Technology}

Wi-Fi signal propagates in the way of non-line-of-sight propagation in complex indoor environments (scattering, diffraction, refraction), causing multipath effects, so it is not sufficient to only use Wi-Fi signal strength to gain positioning results.

As smart phone technology developed, micro sensors such as accelerometers, magnetic compasses, pressure sensors, gyroscopes, etc. have been integrated into these devices, which are generally representative of mobile terminals. Researchers have used micro sensors combined with Wi-Fi fingerprint technology to further improve positioning results. This trend is now mainstream in the indoor positioning field and this technology is widely used.

With this development, assistive positioning technology has been introduced to increase positioning accuracy, to adjust the positioning results and to expand the positioning function, which plays an assistive role in Wi-Fi fingerprint indoor positioning systems. Based on this, we decided to call this kind of approach assistive positioning technology.

\subsection{Indoor Location Based on Inertial Sensor Technology}

Inertial sensors make full use of micro-sensors based on the micro-electro-mechanical system (MEMS), which is a combination of micro-sensors, such as accelerometers, gyroscopes, magnetic compasses, etc. Currently, inertial sensors based on indoor positioning technology are mainly divided into two categories [77]. One is based on Newton's law of motion, and obtains the speed and position of movement by calculating acceleration. Since data drift occurs when this method is used in practice, the cumulative error of multiple calculations can reach tens of meters or even hundreds of meters within a few seconds. Another method is pedestrian dead reckoning (PDR), which uses the number of steps that pedestrians walk to gain results. Due to its high accuracy, it has become an area of much interest in the assisted positioning technology field.

The UnLoc positioning system based on track estimation was designed by Wang [78] at Duke University, and utilizes the accelerometer and magnetic compass combined with dead reckoning. Wang [78] used an accelerometer to judge the number of steps and direction of the moving user, before using relevant techniques to obtain the user's location information.

Zhang [79] used an improved algorithm of PDR. In the PDR algorithm, the traditional methods of calculating steps are the zero-crossing method and peak detection algorithm. Both methods have a fixed threshold value and require a steady speed. The method proposed in reference [79] used dynamic threshold and time window to measure the number of steps. It could self-regulate based on the state of the environment and pedestrians. In the same reference [79], the experimental results showed that the average positioning error of the method could be maintained around $0.57 \mathrm{~m}$, with a maximum positioning error of only $1.4 \mathrm{~m}$. 
The Zee positioning system proposed by Rai et al. [80] used gyroscopes, accelerometers, and electronic compasses to predict the number of steps in combination with particle filtering.

\subsection{Geomagnetic-Based Indoor Positioning Technology}

A dozen years ago, outdoor positioning using geomagnetic methods were proposed and implemented. During the study, it was found that magnetic positioning did not require deploying an additional hardware environment. The data it used were easy to acquire and were less affected by the external environment. In the development of indoor positioning, geomagnetism has gradually attracted people's attention, and has become a research hotspot [81]. Geomagnetic positioning in indoor environments and buildings is mainly influenced by the steel skeleton structure. Li et al. [82] deeply discussed the feasibility of geomagnetic location, and thought that there were still many problems to be solved despite the obvious advantages of geomagnetic location.

Chung et al. [83] used geomagnetic signals as fingerprint data to locate indoor fingerprints. They analyzed the characteristics of geomagnetic data and proposed geomagnetic maps and geomagnetic matching methods based on position and orientation. In reference [83], $90 \%$ of the positioning accuracy could reach $1.64 \mathrm{~m}$ and $50 \%$ could reach $0.71 \mathrm{~m}$.

\subsection{Indoor Positioning Technology Based on Wi-Fi Signal Characteristics}

Abdelnasser et al. [84] analyzed the characteristics of Wi-Fi signals and found that there were common features of Wi-Fi signals in different areas of an indoor environment. Abdelnasser [84] proposed a semantic-based SLAM (Simultaneous Localization and Mapping) positioning method based on these features. Abdelnasser [84] established the landmark distribution of indoor environment based on the sampling information of various micro-sensors. This method clustered the signal strength samples according to the characteristics of Wi-Fi signal strength near different landmarks and then it combined with a SLAM framework for positioning, with the average positioning error reaching $0.53 \mathrm{~m}$.

\section{Miscellaneous}

To examine the effects of the positioning approach, experimental work was necessary. To save on costs and time, there are lots of resources available online.

Collecting data is always the first step of indoor positioning, which is important but cost intensive. Thanks to the researchers, there are lots of public databases available on RSS:

The uiuc/uim dataset: a set of Bluetooth and Wi-Fi traces collected by 28 Google Android phone users (staff, faculties, grads, and undergrads at the University of Illinois) for three weeks in March 2010. Each UIM experimental phone contained Bluetooth and a Wi-Fi scanners to capture both Bluetooth MAC addresses and Wi-Fi access point MAC addresses in the proximity of the phone [85].

The kth/rss dataset: This dataset contains the RSS (Radio Signal Strength) data collected with a mobile robot in two environments: indoor (KTH) and outdoor (Dortmund). RSSI metric was used to collect the RSS data in terms of $\mathrm{dBm}$. The mobile robot location was recorded using its odometry (dead reckoning) [86].

Smartphone Dataset for Human Activity Recognition (HAR) in Ambient Assisted Living (AAL) Data Set: This dataset was used to improve the accuracy of our HAR algorithms applied in a social connectedness experiment in the domain of Ambient Assisted Living. The dataset was collected from the in-built accelerometer and gyroscope of a smart phone worn around the waists of the participants. The data was collected from 30 participants within the age range of 22-79 years. Each activity (standing, sitting, laying, walking, walking upstairs, walking downstairs) was performed for $60 \mathrm{~s}$, and the tri-axial linear acceleration and tri-axial angular velocity were collected at a constant rate of $50 \mathrm{~Hz}$ [87].

UJIIndoorLoc Data Set: The UJIIndoorLoc database covers three buildings of Universitat Jaume I with four or more floors and almost $110.000 \mathrm{~m}^{2}$. It was created in 2013 by means of more than 20 different users and 25 Android devices. The database consists of 19,937 training/reference records 
and 1111 validation/test records. The 529 attributes contain the Wi-Fi fingerprint, the coordinates where it was taken, and other useful information [88].

UJIIndoorLoc-Mag Data Set: The UJIIndoorLoc-Mag database can be used to compare magnetic field-based indoor localization methods. It consists of 270 continuous samples for training and 11 for testing. Each sample comprises a set of discrete captures taken along a corridor (or an intersection) with a period of $0.1 \mathrm{~s}$. In total, there are almost 40,000 discrete captures, where each one contains features obtained from the magnetometer, the accelerometer, and the orientation sensor of the device [89].

There are many top competitions of indoor positioning with many of the competitors' positioning approaches improving the entire indoor positioning field. The most valuable competitions are listed below: Microsoft Indoor Localization Competition @ IPSN (2014-2017) [90], EvAAL Competition (2011-2015) [1], IPIN Competition (2016-2017) [38], and the EVARILOS project and competition [91]. Besides, [92,93] introduced the benchmarking tools for indoor positioning solutions.

\section{Conclusions and Future Work}

In this paper, we discussed indoor Wi-Fi positioning technology, including the various phases and processes of Wi-Fi fingerprinting technology and we classified the methods used across the various phases. We introduced the studies on Wi-Fi fingerprint indoor positioning technology in recent years and discussed the research trends of indoor positioning. We acknowledge that there are still many potential areas in the indoor fingerprint positioning field that can be improved.

1. Integrating different methods to overcome the defects that appeared when methods were used alone. Additionally, optimizing the whole positioning system, and integrating diverse methods in different phases can overcome problems appearing in every phase. Utilizing new methods is the key to finding new ways to optimize positioning systems.

2. Due to the expansion of the positioning environment and the corresponding increase in fingerprint data, traditional ways of capturing fingerprint data are no longer suitable as they are time and labor intensive. Thus, it is necessary to find new ways of obtaining fingerprint data quickly and easily, like crowdsourcing [87].

3. There are still many unresolved problems in Wi-Fi signal due to its disadvantages which can have a bad influence on positioning results. Thus, finding a new physical media or signal that is better than Wi-Fi may be one way to resolve the issue.

According to the three points discussed above, we foresee that Wi-Fi indoor positioning will develop in the following areas:

1. The integration of different methods and utilizing new methods, for example, the integration of deterministic and probabilistic methods. Data are analyzed by probabilistic positioning analysis for the user's historical data, and then the deterministic method is used for accurate positioning.

2. The LIFS positioning systems [4] proposed a new direction in indoor positioning development, which tried to reduce labor consumption in the off-line acquisition phase by using methods like crowdsourcing. This direction is also one of the new developing trends of indoor positioning.

3. In recent years, some studies have started to use information of a physical level instead of RSS to get fingerprint data like channel impulse response (CIR), which has better time stability and spatial sensitivity. Thus, using different physical media [94-96] is also a research pathway.

Indoor positioning has developed considerably in the past decade, but also faces many new challenges and potential problems like high calculating costs, and the accuracy of positioning is not stable and satisfied. The novelty of positioning systems deserves special mention, and is important in this field. The innovation of technical methods and hardware inventions inject new vitality to the indoor positioning market, which has greatly promoted indoor positioning technology development in the past decade. 
Acknowledgments: This work was supported by the State's Key Project of Research and Development Plan (with grant of 2016YFC0600908).

Author Contributions: Shixiong Xia provided some valuable idea and gave the structure of this paper. Yi Liu involved in the writing of the manuscript, drafted the manuscript, and responded to the reviewers' comments. Guan Yuan proposed the research and gave the full instruction to overview indoor positioning. Mingjun Zhu and Zhaohui Wang collected relative references.

Conflicts of Interest: The authors declare no conflict of interest.

\section{References}

1. Sithole, G.; Zlatanova, S. Position, Location, Place and Area: An Indoor Perspective. In Proceedings of the ISPRS Annals of Photogrammetry Remote Sensing \& Spatial Information Sciences, Prague, Czech Republic, 12-19 July 2016; pp. 89-96.

2. Bahl, P.; Padmanabhan, V.N. RADAR: An In-Building RF-based User Location and Tracking System. In Proceedings of the 19th Annual Joint Conference of the IEEE Computer and Communications Societies, Tel Aviv, Israel, 26-30 March 2000; pp. 775-784.

3. Youssef, M.; Agrawala, A. The Horus WLAN location determination system. In Proceedings of the 3rd International Conference on Mobile Systems, Applications, and Services, Seattle, WA, USA, 6-8 June 2005; pp. 205-218.

4. Wu, C.; Yang, Z.; Liu, Y. Smartphones Based Crowdsourcing for Indoor Localization. IEEE Trans. Mob. Comput. 2015, 14, 444-457. [CrossRef]

5. Moreira, A.; Meneses, F. Where@UM—Dependable organic radio maps. In Proceedings of the 2015 International Conference on Indoor Positioning and Indoor Navigation, Banff, AB, Canada, 13-16 October 2015.

6. Li, H. Low-cost 3D bluetooth indoor positioning with least square. Wirel. Pers. Commun. 2014, 78, 1331-1344. [CrossRef]

7. Sugano, M.; Kawazoe, T.; Ohta, Y.; Murata, M. Indoor Localization System using RSSI Measurement of Wireless Sensor Network based on ZigBee Standard. In Proceedings of the 6th IASTED International Multi-Conference on Wireless and Optical Communications, Wireless Sensor Networks, Banff, AB, Canada, 3-5 July 2006.

8. Lee, H.C.; Chang, Y.; Park, G.; Ryu, J.; Jeong, S.-G.; Park, S.; Park, J.W.; Hong, K.-S.; Lee, H.C. Indoor positioning system based on incident angles of infrared emitters. In Proceedings of the 30th Annual Conference of IEEE Industrial Electronics Society, (IECON 2004), Busan, South Korea, 2-6 November 2004.

9. Gigl, T.; Janssen, G.J.M.; Dizdarevic, V.; Witrisal, K.; Irahhauten, Z. Analysis of a UWB indoor positioning system based on received signal strength. In Proceedings of the 4th Workshop on Positioning, Navigation and Communication, (WPNC ‘07), Hannover, Germany, 22 March 2017.

10. Hazas, M.; Hopper, A. Broadband ultrasonic location systems for improved indoor positioning. IEEE Trans. Mob. Comput. 2006, 5, 536-547. [CrossRef]

11. Vo, Q.D.; De, P.A. Survey of Fingerprint-Based Outdoor Localization. IEEE Commun. Surv. Tutor. 2015, $18,1$. [CrossRef]

12. Altintas, B.; Serif, T. Improving RSS-Based Indoor Positioning Algorithm via K-Means Clustering. In Proceedings of the 11th Europian Wireless Conference 2011-Sustainable Wireless Technologies, Berlin, Germany, 27-29 April 2011; pp. 1-5.

13. Deng, Z.-L.; Yu, Y.-P.; Xu, L.-M. Wireless Location and Navigation in Indoor and Outdoor; Beijing University of Posts and Telecommunications Press: Beijing, China, 2013.

14. Roshanaei, M.; Maleki, M. Dynamic-KNN: A novel locating method in WLAN based on Angle of Arrival. In Proceedings of the 2009 IEEE Symposium on Industrial Electronics \& Applications, Kuala Lumpur, Malaysia, 4-6 October 2009; pp. 722-726.

15. Wang, X.; Wang, Z.; O'Dea, B.A. TOA-based location algorithm reducing the errors due to non-line-of-sight (NLOS) propagation. IEEE Trans. Vehic. Technol. 2003, 52, 112-116. [CrossRef]

16. Yamasaki, R.; Ogino, A.; Tamaki, T.; Uta, T.; Matsuzawa, N.; Kato, T. TDOA location system for IEEE 802.11b WLAN. In Proceedings of the 2005 IEEE Wireless Communications and Networking Conference, New Orleans, LA, USA, 13-17 March 2005. 
17. Izquierdo, F.; Ciurana, M.; Barceló, F.; Paradells, M.; Zola, E. Performance evaluation of a TOA-based trilateration method to locate terminals in WLAN. In Proceedings of the 2006 1st International Symposium on Wireless Pervasive Computing, Phuket, Thailand, 16-18 January 2006.

18. Liu, H.; Darabi, H.; Banerjee, P.; Liu, J. Survey of Wireless Indoor Positioning Techniques and Systems. IEEE Trans. Syst. Man Cybern. Part C 2007, 37, 1067-1080. [CrossRef]

19. Yang, R.-M.; Dai, Q.-Y.; Yu, C.-B. Development of Distance-Independent Indoor Path Loss Model. Telecommun. Sci. 2013, 29, 95-99.

20. Bai, Y.B.; Wu, S.; Retscher, G.; Kealy, A.; Holden, L.; Tomko, M.; Borriak, A.; Hu, B.; Wu, H.R.; Zhang, K. A new method for improving Wi-Fi-based indoor positioning accuracy. J. Locat. Based Serv. 2014, 8, 135-147. [CrossRef]

21. Griswold, W.G.; Shanahan, P.; Brown, S.W.; Boyer, R.; Ratto, M.; Shapiro, R.B.; Truong, T.M. ActiveCampus: Experiments in community-oriented ubiquitous computing. Computer 2004, 37, 73-81. [CrossRef]

22. Chen, L.-N. WLAN Location Fingerprint Indoor Location Key Technology Research; East China Normal University: Shanghai, China, 2014.

23. Wu, C.; Yang, Z.; Liu, Y.; Xi, W. WILL: Wireless Indoor Localization without Site Survey. IEEE Trans. Parallel Distrib. Syst. 2013, 24, 64-72.

24. Zhuang, Y.; Syed, Z.; Georgy, J.; El-Sheimy, N. Autonomous smartphone-based WiFi positioning system by using access points localization and crowdsourcing. Pervasive Mob. Comput. 2015, 18, 118-136. [CrossRef]

25. Mautz, R. Indoor Positioning Technologies. SüdwestdeutscherVerlagfürHochschulschriften 2012, 2, $15-24$.

26. Farid, Z.; Nordin, R.; Ismail, M. Recent Advances in Wireless Indoor Localization Techniques and System. J. Comput. Netw. Commun. 2013, 2013, 15. [CrossRef]

27. Garcia-Villalonga, S.; Perez-Navarro, A. Influence of human absorption of Wi-Fi signal in indoor positioning with Wi-Fi fingerprinting. In Proceedings of the 2015 International Conference on Indoor Positioning and Indoor Navigation (IPIN), Banff, AB, Canada, 13-16 October 2015.

28. Walters, C. Characterization of Smart Phone Received Signal Strength Indication for WLAN Indoor Positioning Accuracy Improvement. J. Netw. 2014, 9, 1061-1065.

29. Song, J.; Hur, S.; Park, Y.; Choi, J. An improved RSSI of geomagnetic field-based indoor positioning method involving efficient database generation by building materials. In Proceedings of the 2016 International Conference on Indoor Positioning and Indoor Navigation (IPIN), Alcala de Henares, Spain, 4-7 October 2016.

30. Fang, S.H.; Lin, T.N.; Lee, K.C. A Novel Algorithm for Multipath Fingerprinting in Indoor WLAN Environments. IEEE Trans. Wirel. Commun. 2008, 7, 3579-3588. [CrossRef]

31. Kaemarungsi, K.; Krishnamurthy, P. Properties of Indoor Received Signal Strength for WLAN Location Fingerprinting. In Proceedings of the 1st Annual International Conference on Mobile and Ubiquitous Systems: Networking and Services (MOBIQUITOUS 2004), Boston, MA, USA, 22-26 August 2004.

32. Moghtadaiee, V.; Dempster, A.G. Design protocol and performance analysis of indoor fingerprinting positioning systems. Phys. Commun. 2014, 13, 17-30. [CrossRef]

33. Moghtadaiee, V.; Dempster, A.G. WiFi fingerprinting signal strength error modeling for short distances. In Proceedings of the 2012 International Conference on Indoor Positioning and Indoor Navigation, Sydney, Australia, 13-15 November 2012.

34. Swangmuang, N.; Krishnamurthy, P.V. On clustering RSS fingerprints for improving scalability of performance prediction of indoor positioning systems. In Proceedings of the 1st ACM International Workshop on Mobile Entity Localization and Tracking in Gps-Less Environments, San Francisco, CA, USA, 19 September 2008; pp. 61-66.

35. Jung, S.H.; Moon, B.-C.; Han, D. Performance Evaluation of Radio map Construction Methods for Wi-Fi Positioning Systems. IEEE Trans. Intell. Transp. Syst. 2016, 18, 880-889. [CrossRef]

36. Liu, W.; Fu, X.; Deng, Z.; Xu, L.; Jiao, J. Smallest enclosing circle-based fingerprint clustering and modified-WKNN matching algorithm for indoor positioning. In Proceedings of the 2016 International Conference on Indoor Positioning and Indoor Navigation, Alcala de Henares, Spain, 4-7 October 2016.

37. Fetzer, T.; Ebner, F.; Deinzer, F.; Köping, L.; Grzegorzek, M. On Monte Carlo Smoothing in Multi Sensor Indoor Localisation. In Proceedings of the International Conference on Indoor Positioning and Indoor Navigation (IPIN), Madrid, Spain, 4-7 October 2016. 
38. Torres-Sospedra, J.; Jiménez, A.R.; Knauth, S.; Moreira, A.; Beer, Y.; Fetzer, T.; Ta, V.-C.; Montoliu, R.; Seco, F.; Mendoza-Silva, G.M.; et al. The Smartphone-Based Offline Indoor Location Competition at IPIN 2016: Analysis and Future Work. Sensors 2017, 17, 557. [CrossRef] [PubMed]

39. Torres-Sospedra, J.; Moreira, A.; Knauth, S.; Berkvens, R.; Montoliu, R.; Belmonte, O.; Trilles, S.; João, N.M.; Meneses, F.; Costa, A.; et al. A Realistic Evaluation of Indoor Positioning Systems Based on Wi-Fi Fingerprinting: The 2015 EvAAL-ETRI Competition. J. Ambient Intell. Smart Environ. 2017, 9, 263-279. [CrossRef]

40. Zhang, W.; Hua, X.; Yu, K.; Qiu, W.; Zhang, S. Domain clustering based WiFi indoor positioning algorithm. In Proceedings of the 2016 International Conference on Indoor Positioning and Indoor Navigation (IPIN), Alcala de Henares, Spain, 4-7 October 2016.

41. Berkvens, R.; Weyn, M.; Peremans, H. Position error and entropy of probabilistic Wi-Fi fingerprinting in the UJIIndoorLoc dataset. In Proceedings of the 2016 International Conference on Indoor Positioning and Indoor Navigation (IPIN), Alcala de Henares, Spain, 4-7 October 2016.

42. Mizmizi, M.; Reggiani, L. Design of RSSI based fingerprinting with reduced quantization measures. In Proceedings of the 2016 International Conference on Indoor Positioning and Indoor Navigation (IPIN), Madrid, Spain, 4-7 October 2016.

43. Kochláň, M.; Miček, J. Indoor propagation of 2.4 GHz radio signal propagation models and experimental results. In Proceedings of the 10th International Conference on Digital Technologies 2014, Žilina, Slovakia, 9-11 July 2014; pp. 125-129.

44. Kaemarungsi, K.; Krishnamurthy, P. Analysis of WLAN's received signal strength indication for indoor location fingerprinting. Pervasive Mob. Comput. 2012, 8, 292-316. [CrossRef]

45. Seidel, S.Y.; Rappaport, T.S. $914 \mathrm{MHz}$ path loss prediction models for indoor wireless communications in multi-floored-buildings. IEEE Trans. Antennas Propag. 1992, 40, 207-217. [CrossRef]

46. Nurminen, H.; Talvitie, J.; Ali-Löytty, S.; Müller, P.; Lohan, E.-S.; Piché, R.; Renfors, M. Statistical path loss parameter estimation and positioning using RSS measurements in indoor wireless networks. In Proceedings of the 2012 International Conference on Indoor Positioning and Indoor Navigation (IPIN), Sydney, Australia, 13-15 November 2012.

47. Laitinen, E.; Lohan, E.-S. On the Choice of Access Point Selection Criterion and Other Position Estimation Characteristics for WLAN-Based Indoor Positioning. Sensors 2016, 16, 737. [CrossRef] [PubMed]

48. Teramoto, Y.; Asahara, A. Wireless LAN based indoor positioning using radio-signal strength distribution modeling. In Proceedings of the 2012 International Conference on Indoor Positioning and Indoor Navigation (IPIN), Sydney, Australia, 13-15 November 2012.

49. Lim, H.; Kung, L.-C.; Hou, J.C.; Luo, H. Zero-Configuration, Robust Indoor Localization: Theory and Experimentation. Proc.-IEEE INFOCOM 2005, 268, 229-236.

50. Lee, C.-W.; Lin, T.-N.; Fang, S.-H.; Chou, Y.-C. A novel clustering-based approach of indoor location fingerprinting. In Proceedings of the 2013 IEEE 24th Annual International Symposium on Personal, Indoor, and Mobile Radio Communications (PIMRC), London, UK, 8-11 September 2013.

51. Song, C.; Wang, J.; Yuan, G. Hidden Naive Bayes Indoor Fingerprinting Localization Based on Best-Discriminating AP Selection. ISPRS Int. J. Geo-Inf. 2016, 5, 189. [CrossRef]

52. Jhuang, F.-M.; Hung, C.-F.; Tuan, C.-C.; Wu, Y.-C.; Leu, F.-Y. An AP Selection with RSS Standard Deviation for Indoor Positioning in Wi-Fi. In Proceedings of the 2015 9th International Conference on Innovative Mobile and Internet Services in Ubiquitous Computing (IMIS), Blumenau, Brazil, 8-10 July 2015.

53. Feng, C. Research and Implementation of RSS Indoor Location System Based on Compression Sensing; Beijing Jiaotong University: Beijing, China, 2010.

54. Ma, J.; Li, X.; Tao, X.; Lu, J. Cluster filtered KNN: A WLAN-based indoor positioning scheme. In Proceedings of the 2008 International Symposium on a World of Wireless, Mobile and Multimedia Networks, 2008 (WoWMoM 2008), Newport Beach, CA, USA, 23-26 June 2008.

55. Guowei, Z.; Zhan, X.; Dan, L. Research and improvement on indoor localization based on RSSI fingerprint database and K-nearest neighbor points. In Proceedings of the 2013 International Conference on Communications, Circuits and Systems (ICCCAS), Chengdu, China, 15-17 November 2013.

56. Shin, B.; Lee, J.H.; Lee, T.; Kim, H.S. Enhanced weighted K-nearest neighbor algorithm for indoor Wi-Fi positioning systems. In Proceedings of the 2012 8th International Conference on Computing Technology and Information Management (ICCM), Seoul, Korea, 24-26 April 2012. 
57. Brunato, M.; Battiti, R. Statistical learning theory for location fingerprinting in wireless LANs. Comput. Netw. 2002, 47, 825-845. [CrossRef]

58. Abdou, A.S.; Aziem, M.A.; Aboshosha, A. An efficient indoor localization system based on Affinity Propagation and Support Vector Regression. In Proceedings of the 2016 Sixth International Conference on Digital Information Processing and Communications (ICDIPC), Beirut, Lebanon, 21-23 April 2016.

59. Wu, Z.; Liu, J.; Liu, B. Particle Filter and Support Vector Machine Based Indoor Localization System, Microsoft Indoor Localization Competition-IPSN 2016. Available online: https://www.microsoft.com/enus/research/wp-content/uploads/2015/10/zhengwu.pdf/ (accessed on 12 April 2017).

60. Dai, H.; Ying, W.; Xu, J. Multi-layer neural network for received signal strength-based indoor localisation. IET Commun. 2016, 10, 717-723. [CrossRef]

61. Fang, S.H.; Lin, T.N. Indoor location system based on discriminant-adaptive neural network in IEEE 802.11 environments. IEEE Trans. Neural Netw. 2008, 19, 1973-1978. [CrossRef] [PubMed]

62. Battiti, R.; Le, N.T.; Villani, A. Location-Aware Computing: A Neural Network Model for Determining Location in Wireless LANs; University of Trento: Trento, Italy, 2002.

63. Nowicki, M.; Wietrzykowski, J. Low-Effort Place Recognition with Wi-Fi Fingerprints Using Deep Learning; Springer International Publishing: Cham, Switzerland, 2016.

64. Kushki, A.; Plataniotis, K.N.; Venetsanopoulos, A.N. Kernel-based positioning in wireless local area networks. IEEE Trans. Mob. Comput. 2007, 6, 689-705. [CrossRef]

65. Yim, J. Introducing a decision tree-based indoor positioning technique. Expert Syst. Appl. 2008, 34, $1296-1302$. [CrossRef]

66. Kontkanen, P.; Myllymäki, P.; Roos, T.; Tirri, H.; Valtonen, K.; Wettig, H. Topics in probabilistic location estimation in wireless networks. In Proceedings of the 15th IEEE International Symposium on Personal, Indoor and Mobile Radio Communications, Barcelona, Spain, 5-8 September 2014.

67. Luo, J.; Zhan, X. Characterization of smart phone received signal strength indication for WLAN indoor positioning accuracy improvement. J. Netw. 2014, 9, 739-746. [CrossRef]

68. Chen, M. Study on WLAN Indoor Location and Tracking System Based on Signal Strength; Wuhan University: Wuhan, China, 2012.

69. Ferris, B.; Haehnel, D.; Fox, D. Gaussian processes for signal strength-based location estimation. In Proceedings of the Robotics: Science and Systems, Philadelphia, PA, USA, 16-19 August 2006.

70. Bisio, I.; Lavagetto, F.; Marchese, M.; Sciarrone, A. Smart probabilistic fingerprinting for Wi-Fi-based indoor positioning with mobile devices. Pervasive Mob. Comput. 2016, 31, 107-123. [CrossRef]

71. Madigan, D.; Elnahrawy, E.; Martin, R.P.; Ju, W.-H.; Krishnan, P.; Krishnakumar, A.S. Bayesian indoor positioning systems. In Proceedings of the IEEE 24th Annual Joint Conference of the IEEE Computer and Communications Societies, Piscataway, NJ, USA, 13-17 March 2005; Volume 2, pp. 1217-1227.

72. Patwari, N.; Hero, A.O.; Perkins, M.; Correal, N.S.; O'Dea, R.J. Relative location estimation in wireless sensor networks. IEEE Trans. Signal Process. 2003, 51, 2137-2148. [CrossRef]

73. Honkavirta, V. Location Fingerprinting Methods in Wireless Local Area Networks. Master's Thesis, Tampere University of Technology, Tampere, Finland, October 2008.

74. Gordon, N.; Ristic, B.; Arulampalam, S. Beyond the Kalman Filter: Particle Filters for Tracking Applications; Artech House: London, UK, 2004.

75. Wu, Y. Indoor Positioning Technology and It's Filtering and Fusion Algorithm; North China University of Technology: Beijing, China, 2014.

76. Zhang, S.-Z. Design and Implementation of Indoor Positioning System Based on Inertial Sensor and Wi-Fi; Beijing University of Posts and Telecommunications: Beijing, China, 2012.

77. Wang, H.; Sen, S.; Elgohary, A.; Farid, M.; Youssef, M.; Choudhury, R.R. No need to war-drive: Unsupervised indoor localization. In Proceedings of the 10th International conference on Mobile systems, applications, and services, Lake District, UK, 25-29 June 2012.

78. Zhang, M.; Shen, W.; Yao, Z.; Zhu, J. Multiple information fusion indoor location algorithm based on Wi-Fi and improved. In Proceedings of the 35th Chinese Control Conference (CCC), Chengdu, China, 27-29 July 2016.

79. Rai, A.; Chintalapudi, K.K.; Padmanabhan, V.N.; Sen, R. Zee: Zero-effort crowdsourcing for indoor localization. In Proceedings of the 18th Annual International Conference on Mobile Computing and Networking, Istanbul, Turkey, 22-26 August 2012. 
80. Ettlinger, A.; Retscher, G. Positioning using ambient magnetic fields in combination with Wi-Fi and RFID. In Proceedings of the International Conference on Indoor Positioning and Indoor Navigation, Alcala de Henares, Spain, 4-7 October 2016.

81. Li, B.; Gallagher, T.; Dempster, A.G.; Rizos, C. How feasible is the use of magnetic field alone for indoor positioning? In Proceedings of the 2012 International Conference on. IEEE Indoor Positioning and Indoor Navigation (IPIN), Sydney, Australia, 13-15 November 2012.

82. Chung, J.; Donahoe, M.; Schmandt, C.; Kim, I.-J.; Razavai, P.; Wiseman, M. Indoor location sensing using geo-magnetism. In Proceedings of the 9th International Conference on Mobile Systems, Applications, and Services, Bethesda, MD, USA, 28 June-1 July 2011; pp. 141-154.

83. Abdelnasser, H.; Mohamed, R.; Elgohary, A.; Alzantot, M.F.; Wang, H.; Sen, S.; Choudhury, R.R.; Youssef, M. SemanticSLAM: Using environment landmarks for unsupervised indoor localization. IEEE Trans. Mob. Comput. 2016, 15, 1770-1782. [CrossRef]

84. Nahrstedt, K.; Vu, L. CRAWDAD Dataset uiuc/uim (v. 2012-01-24); University of Illinois Movement (UIM): Champaign, IL, USA, 2012.

85. Ramviyas, P.; Sergio, C.; Fredrik, B.; Petter, O. CRAWDAD Dataset kth/rss (v. 2016-01-05); Computer Vision and Active Perception Lab., Centre for Autonomous Systems, School of Computer Science and Communication, Royal Institute of Technology (KTH): Stockholm, Sweden, 2016.

86. Smartphone Dataset for Human Activity Recognition (HAR) in Ambient Assisted Living (AAL) Data Set; University of Genova, Department of Electrical, Electronic, Telecommunications Engineering and Naval Architecture (DITEN): Genova, Italy, 2016.

87. Torres-Sospedra, J.; Montoliu, R.; Martínez-Usó, A.; Arnau, T.J.; Avariento, J.P.; Benedito-Bordonau, M.; Huerta, J. UJIIndoorLoc: A New Multi-building and Multi-floor Database for WLAN Fingerprint-based Indoor Localization Problems. In Proceedings of the Fifth International Conference on Indoor Positioning and Indoor Navigation, Busan, Korea, 27-30 October 2014.

88. Torres-Sospedra, J.; Rambla, D.; Montoliu, R.; Belmonte, O.; Huerta, J. UJIIndoorLoc-Mag: A New Database for Magnetic Field-Based Localization Problems. In Proceedings of the Sixth International Conference on Indoor Positioning and Indoor Navigation (IPIN 2015), Banff, AB, Canada, 13-16 October 2015.

89. Microsoft Indoor Localization Competition-IPSN 2016. Available online: https://www.microsoft.com/enus/research/event/microsoft-indoor-localization-competition-ipsn-2016/ (accessed on 12 April 2017).

90. EVARILOS project. Available online: http:/ / www.evarilos.eu (accessed on 12 April 2017).

91. Haute, T.V.; Poorter, E.D.; Lemic, F.; Handziski, V.; Wirström, N.; Wolisz, A.; Moerman, I. Demonstration Abstract: Platform for Benchmarking RF-Based Indoor Localization Solutions. In Proceedings of the ACM/IEEE International Conference on Information Processing in Sensor Networks, Vienna, Austria, 11-14 April 2016.

92. Lemic, F.; Buesch, J.; Chwalisz, M.; Handziski, V.; Wolisz, A. Demo Abstract: Testbed Infrastructure for Benchmarking RF-based Indoor Localization Solutions under Controlled Interference. In Proceedings of the European Conference on Wireless Sensor Networks 2014, Oxford, UK, 17-19 February 2014.

93. Zhang, D.; Qiu, G.; Gao, Y.; Fang, X.; Cheng, R.; Chang, A.; Chan, C.-Y. Crowdsourcing based radio map anomalous event detection system for calibration-on-demand. In Proceedings of the 2014 International Conference on Indoor Positioning and Indoor Navigation, Busan, Korea, 27-30 October 2014.

94. Zhang, Y.-C. Research and Implementation of Hybrid Indoor Trajectory Tracking Technology Based on Wi-Fi; Shanghai Jiaotong University: Shanghai, China, 2015.

95. Ochiai, M.; Fujii, M.; Ito, A.; Watanabe, Y.; Hatano, H. A study on indoor position estimation based on fingerprinting using GPS signals. In Proceedings of the 2014 International Conference on Indoor Positioning and Indoor Navigation, Busan, Korea, 27-30 October.

96. Aloui, N.; Raoof, K.; Bouallegue, A.; Letourneur, S.; Zaibi, S. A novel indoor localization scheme based on fingerprinting technique and CDMA signals. In Proceedings of the 2012 International Conference on Indoor Positioning and Indoor Navigation (IPIN), Sydney, Australia, 13-15 November 2012.

(C) 2017 by the authors. Licensee MDPI, Basel, Switzerland. This article is an open access article distributed under the terms and conditions of the Creative Commons Attribution (CC BY) license (http:/ / creativecommons.org/licenses/by/4.0/). 